\title{
Experimental Investigation of Microwave Imaging via Space-Time Beamforming for Breast Cancer Detection
}

\author{
Xu Li, Susan C. Hagness, Barry D. Van Veen, and Daniel van der Weide \\ Department of Electrical and Computer Engineering, University of Wisconsin-Madison \\ Madison, WI 53706, United States
}

\begin{abstract}
We investigate the experimental feasibility of detecting small malignant breast tumors using a recently proposed method of microwave imaging via space-time beamforming. A microwave sensor comprised of a planar synthetic array of compact ultrawideband (1-11 GHz) antennas is placed above a breast tissue phantom - a tank of homogeneous normal breast tissue simulant covered by a thin layer of skin simulant. A small $(<0.5 \mathrm{~cm})$ synthetic tumor is embedded in the breast phantom. At each position in the array, the antenna transmits a synthetically generated ultra-short pulse into the breast phantom. A robust data-adaptive algorithm removes the artifact caused by the dominant backscatter from the skin-breast interface. The signals are passed through a 3-D space-time beamformer designed to image backscattered energy as a function of location. Even millimeter-sized malignant tumors have relatively large microwave scattering crosssections due to their significant dielectric-properties contrast with normal breast tissue. Therefore they produce localized regions of large backscatter energy levels in the beamformer image. The successful detection and localization of very small synthetic tumors embedded in the skin-breast tissue phantom is demonstrated.
\end{abstract}

\section{INTRODUCTION}

$\mathrm{X}$-ray mammography is the primary method used in breast cancer screening. Although it provides highresolution images at low radiation doses, its limitations are well recognized [1]. Approximately $15 \%$ of all breast cancers are missed by conventional mammography while $75 \%$ of identified breast lesions turn out to be benign. The need for improved sensitivity and specificity, particularly in the case of radiographically dense breast tissue, motivates the search for new technologies.

Several active microwave imaging techniques have been proposed as complementary modalities to X-ray mammography. The underlying principle of microwave imaging is the significant dielectric-properties contrast between malignant tumors and normal breast tissue at microwave frequencies [2]-[4]. This contrast, which is estimated to be greater than $2: 1$, is significantly higher than the few-percent contrast in radiographic density exploited by $\mathrm{X}$-rays and thus provides a compelling rationale for the development of microwave breast cancer detection technology.
We have recently proposed a method of microwave imaging via space-time (MIST) beamforming for breast cancer detection [5]. The space-time beamformer assumes that each antenna in an array sequentially transmits a lowpower ultrawideband (UWB) signal into the breast and records the backscatter. The beamformer spatially focuses the backscatter signals to discriminate against clutter generated by the heterogeneity of normal breast tissue. In contrast to narrowband tomography [6]-[8], the MIST beamforming approach identifies the presence and location of malignant breast tumors by imaging backscatter signal energy as a function of location, rather than reconstructing the dielectric-properties profile of the breast. Thus, through the use of robust signal processing techniques, our approach avoids the computational challenges associated with solving the nonlinear inverse scattering problem.

We previously demonstrated the theoretical feasibility of MIST beamforming by applying 2-D space-time beamformers to simulated backscatter data obtained from a variety of anatomically realistic MRI-derived FDTD breast models [5]. In those studies, the MIST approach was shown to be exceptionally robust with respect to a number of potential challenges associated with imaging the inherently heterogeneous breast. Our recent work [5] has also shown that MIST beamforming offers significant improvement in performance over previous UWB radar techniques based on simpler focusing schemes [9]-[11].

In [12], we presented results from a first-generation breast phantom consisting of a water-based synthetic tumor suspended in a homogeneous normal breast tissue simulant without skin. In this study, we investigate the experimental feasibility of this method using a class of second-generation phantoms which feature a thin layer of skin simulant and normal and malignant tissue simulants that more closely mimic the dielectric-properties contrast observed in actual breast tissue at microwave frequencies.

\section{EXPERIMENTAL DESIGN AND METHODS}

The experimental setup is designed to emulate the clinical configuration where a 2-D antenna array is placed 
near the surface of the naturally flattened breast of a patient lying in a supine position. As shown in Fig. 1, our simple breast phantom consists of a container, filled with a normal breast tissue simulant. In the present experiments, the breast-mimicking medium is homogeneous, thereby representing a volume average of the fat and glandular parenchyma. A thin layer of material representing skin is placed on top of the breast-mimicking material A small synthetic tumor is suspended in the breast phantom.

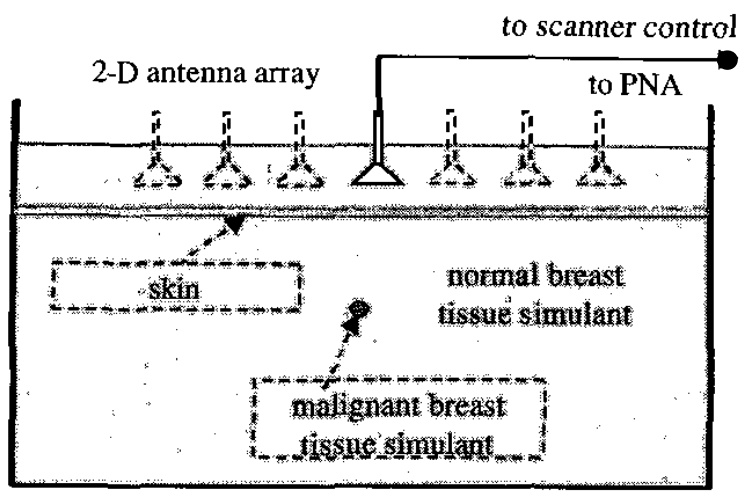

Fig. 1. Schematic showing a side view of the experimental setup.

Soybean oil is an inexpensive, non-toxic liquid with dielectric properties similar to very low-water-content fatty tissue. For this reason, it is used as the normal breast tissue simulant in our preliminary second-generation phantom. The solid-line curves in Fig. 2 show the measured dielectric constant and conductivity in the frequency range of $1 \mathrm{GHz}$ to $11 \mathrm{GHz}$ using an open-ended coaxial probe technique [13]. We note that the dielectric properties of the oil $\left(\varepsilon_{r}=2.6, \sigma=0.05 \mathrm{~S} / \mathrm{m}\right.$ at $\left.6 \mathrm{GHz}\right)$ fall slightly below the expected range of the dielectric constant for fatty breast tissue. Therefore, we have chosen materials for the skin and tumor simulants that similarly underestimate the actual dielectric properties of those tissue types, so that the dielectric-properties contrasts in the breast phantom are more representative of those for actual tissue.

The synthetic tumor is made from a diacetin solution $\left(\varepsilon_{r}=8.7, \sigma=1.9 \mathrm{~S} / \mathrm{m}\right.$ at $6 \mathrm{GHz}$ ). This yields a contrast in dielectric constant between the malignant and normal tis sue simulants of 3.3:1 at $6 \mathrm{GHz}$. The diacetin solution is poured into a 4-mm-diameter cylindrical container that has a height of $4 \mathrm{~mm}$. The dielectric properties of the cylindrical container are similar to those of the soybean oil. The synthetic tumor is suspended in the oil with 0.1-mmdiameter nylon thread.

A 1.5-mm-thick unclad FR4 glass epoxy PC board is used to represent the layer of skin. According to the manufacturer, the dielectric properties of FR4 at $1 \mathrm{GHz}$ are $\varepsilon_{r}=4.34$ and $\tan \delta=0.016$. Thus the dielectric constant of the skin simulant falls in between that of the normal and malignant tissue simulants, as desired.

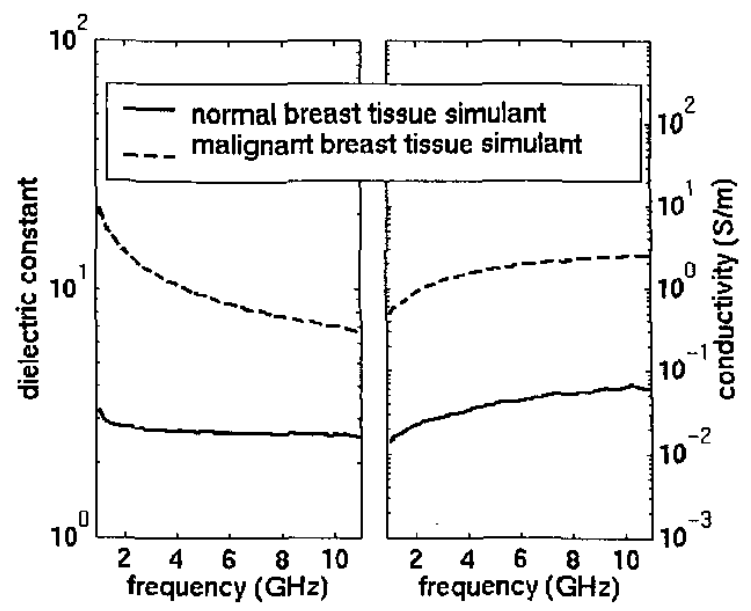

Fig. 2. Measured dielectric properties of the normal and malignant tissue simulants.

To ensure ease of movement in scanning the antenna, the microwave antenna-array sensor is placed $1 \mathrm{~cm}$ above the skin. To reduce reflections from the skin and thereby more efficiently couple energy into the breast, the antenna array is immersed in a matching medium. Here, for simplicity, we use the same soybean oil used as the normal breast tissue simulant. A single UWB antenna is sequentially repositioned using a computer-controlled mechanical x-y scanner to synthesize a 49-element (7 7) antenna array. The synthetic array has a span of $6 \mathrm{~cm}$ in both the $\mathrm{x}$ and $\mathrm{y}$ directions. The antenna is connected to an Agilent E8364A (10 MHz - 50 GHz) performance network analyzer (PNA) to transmit and receive microwave signals at each antenna location.

The UWB antenna is adapted from a design proposed in [14]. As shown in Fig. 3(a), the brass antenna has a pyramidal-horn-shaped ground plane connected to the outer conductor of the coaxial feed via an SMA connector. The height of the pyramidal horn is $1.3 \mathrm{~cm}$ and the aperture dimensions are $2.5 \mathrm{~cm}$ by $2.0 \mathrm{~cm}$. This ground plane configuration confines the main beam of the radiation pattern to the opening of the horn to ensure minimum interference from undesired sources. A curved launching plane is connected to the inner conductor of the SMA connector and loaded with a $50-\Omega$ resistor to suppress antenna reverberations. A metallic ridge structure is attached to the interior side of the horn-shaped ground plane opposite the curved launching plane to further enhance the bandwidth performance of the antenna. Fig. 
3(b) shows that the measured VSWR for the antenna submerged in the immersion medium (soybean oil) is no greater than 1.5 over the entire frequency range of interest $(1-11 \mathrm{GHz})$.
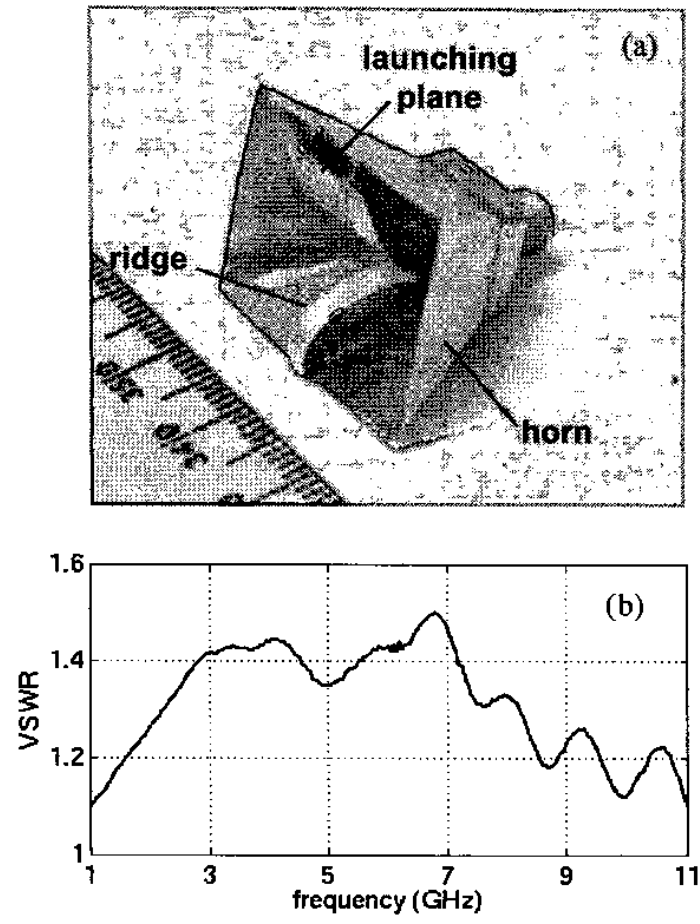

Fig. 3. (a) UWB antenna used in the microwave imaging system. (b) Measured VSWR of the antenna immersed in a medium matched to the normal breast tissue simulant.

At each antenna location in the synthetic array, the PNA performs a frequency sweep from 1 to $11 \mathrm{GHz}$ with 201 frequency samples and records the backscatter $\left(S_{11}\right)$. The frequency-domain backscattered signals are scaled by the spectrum of the desired input pulse and transformed to the time domain using an inverse FFT algorithm. The timedomain input pulse is chosen to be a 135-ps Gaussian pulse modulating a $6-\mathrm{GHz}$ carrier. The sampling rate of the synthesized time-domain backscatter waveforms is $50 \mathrm{GHz}$.

\section{MIST BEAMFORMING RESULTS}

The tumor response in the received waveforms is completely masked by the dominant near-field artifacts including antenna reverberation and reflections from the skin interface. Therefore, we first apply the data-adaptive artifact-removal algorithm reported in Ref. [5] which estimates the artifact in each received waveform as a filtered combination of the signals at all other antenna locations and removes it from the received waveform. The filter weights are chosen to minimize the residual signal mean-squared error calculated over the artifact-dominated early-time response.

The processed waveforms are passed through the space-time beamformer to image backscatter energy as a function of position. First, at each candidate location in the reconstruction region, the processed backscatter waveforms are time-shifted to align the return from a hypothesized point-scatterer at that location. The imaging results presented in this paper are obtained by using the frequency-domain FIR filter design proposed in [15] to obtain a set of beamformers that equalize frequencydependent propagation effects. After the artifact-removal and time alignment steps, the received waveforms are transformed to the frequency domain using an FFT algorithm and passed through the bank of frequencydomain beamformers. The beamformer outputs are then summed, transformed back to the time domain, and timegated. Finally the backscattered energy is calculated. By appropriately changing the time shifts, gating, and FIR filter weights, the beamformer focus is scanned throughout the breast region to obtain a 3-D image of backscattered energy as a function of location. Locations of high energy indicate the presence of strong scatterers, that is, malignant tumors, in the breast.

Fig. 4 shows the MIST beamforming results for the breast phantom of Fig. 1, which contains a 4-mm-diameter synthetic tumor located approximately $2 \mathrm{~cm}$ below the skin surface. The 3-D image of backscatter energy is generated for a $6-\mathrm{cm} 6-\mathrm{cm} 5-\mathrm{cm}$ domain with a $1 \mathrm{~mm}$ pixel resolution. Three orthogonal planes from the 3 -D image are labeled using $\mathrm{x}$ and $\mathrm{y}$ axes that correspond to the lateral dimensions of imaging domain and $a$ axis that corresponds to the depth dimension. The origin of the $z$ axis roughly corresponds to the location of the skin layer. The two energy peaks in the depth direction correspond to scattering from the top and bottom surfaces of the cylindrical tumor. The peak energy nearest the surface is located within $2 \mathrm{~mm}$ of the top edge of the actual tumor. For comparison purposes, the same beamforming process is also applied to the backscatter waveforms obtained from a tumor-free phantom. The signal-to-clutter ratio, defined as the ratio of the estimated tumor energy to the maximum clutter energy in the tumor-free phantom, is $8.4 \mathrm{~dB}$.

\section{CONCLUSIONS}

We have demonstrated the experimental feasibility of the 3-D MIST beamforming approach $[5,15]$ by successfully detecting and imaging backscattered energy from a 4-mmdiameter synthetic tumor in a second-generation breast tissue phantom. The efficacy of the artifact-removal 
algorithm [5] is also fully demonstrated since the phantom includes a skin simulant. These experimental results suggest that microwave imaging via space-time beamforming offers the potential for detecting small breast tumors using state-of-the-art but readily available hardware, UWB antennas, and robust signal processing algorithms.

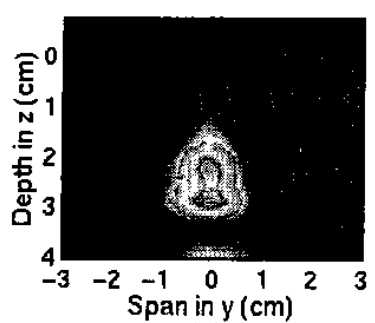

(a)

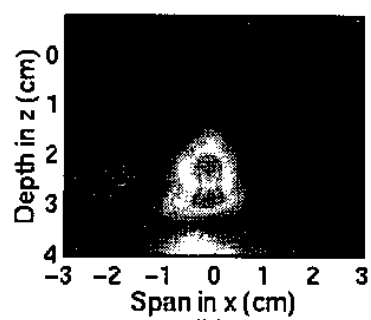

(b)

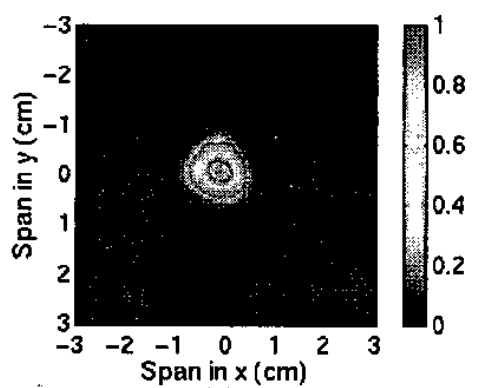

(c)

Fig. 4. Color image of backscattered energy for the breast phantom of Fig. 1, which contains a $4 \mathrm{~mm}$-diameter synthetic tumor located at a depth of $2 \mathrm{~cm}$ below the skin surface. The orthogonal planes shown intersect the shallower of the two energy peaks of the tumor response: (a) yz plane at $\mathrm{x}=-0.1 \mathrm{~cm}$. (b) $x z$ plane at $y=-0.1 \mathrm{~cm}$. (c) $x y$ plane at $z=2.2 \mathrm{~cm}$.

\section{ACKNOWLEDGEMENT}

We thank S. Davis and E. Bond for their assistance in signal processing. We also thank M. Choi, P. Gustafson, and L. Palmer for their help with the experimental setup. This work was supported by the Department of Defense Breast Cancer Research Program under award DAMD1702-1-0625, National Science Foundation BES-0201880, and the National Institutes of Health under grant R21 CA9218801 awarded by the National Cancer Institute.

\section{REFERENCES}

[1] "Mammography and Beyond: Developing Techniques for the Early Detection of Breast Cancer," Inst. of Medicine, National Academy Press, Washington D.C., 2000.
[2] A. J. Surowiec, S. S. Stuchly, J. R. Barr, and A. Swarup, "Dielectric properties of breast carcinoma and the surrounding tissues," IEEE Trans. Biomed. Eng., vol. 35, pp. 257-263, April 1988.

[3] W. T. Joines, Y. Zhang, C. Li, and R. L. Jirtle. "The measured electrical properties of normal and malignant human tissues from 50 to $900 \mathrm{MHz}, "$ Med. Phys., vol. 21, pp. 547-550, April 1994.

[4] S. S. Chaudhary, R. K. Mishra, A. Swarup, and J. M. Thomas, "Dielectric properties of normal and malignant human breast tissues at radiowave and microwave frequencies," Indian J. Biochem. and Biophys., vol. 21, pp. 76-79, Feb. 1984.

[5] E. J. Bond, Xu Li, S. C. Hagness, and B. D. Van Veen, "Microwave imaging via space-time beamforming for early detection of breast cancer," IEEE Trans. Antennas and Propagat., in press.

[6] P. M. Meaney, M. W. Fanning, D. Li, S. P. Poplack, and K. D. Paulsen, "A clinical prototype for active microwave imaging of the breast," IEEE Trans. Microwave Theory Tech., vol. 48, no. 11, pp. 1841-1853, Nov. 2000.

[7] Q. H. Liu, Z. Q. Zhang, T. T. Wang, J. A. Bryan, G. A. Ybarra, L. W. Nolte, and W. T. Joines, "Active microwave imaging I. 2-D forward and inverse scattering methods," IEEE Trans. Microwave Theory Tech., vol. 50, no. 1, pp. 123-133, Jan. 2002.

[8] A. E. Bulyshev, S. Y. Semenov, A. E. Souvorov, R.H. Svenson, A.G. Nazarov, Y. E. Sizov, and G. P. Tatsis, "Computational modeling of three-dimensional microwave tomography of breast cancer," IEEE Trans. Biomed. Eng., vol. 48, pp. 1053-1056, Sept. 2001.

[9] S. C. Hagness, A. Taflove, and J. E. Bridges, "Twodimensional FDTD analysis of a pulsed microwave confocal system for breast cancer detection: Fixed-focus and anternaarray sensors," IEEE Trans. Biomed. Eng., vol. 45, pp. 1470-1479, Dec. 1998.

[10] X. Li and S. C. Hagness, "A confocal microwave imaging algorithm for breast cancer detection," IEEE Microwave Wireless Comp. Lett., vol. 11, pp. 130-132, March 2001.

[11] E. C. Fear, Xu Li, S. C. Hagness, and M. A. Stuchly, "Confocal microwave imaging for breast cancer detection: localization of tumors in three dimensions," IEEE Trans. Biomed. Eng., Vol. 49, no. 8, pp. 812-822, Aug. 2002.

[12] X. Li, E. J. Bond, S. C. Hagness, B. D. Van Veen, and D. van der Weide, "Three-dimensional microwave imaging via space-time beamforming for breast cancer detection," IEEE AP-S International Symposium and USNC/URSI Radio Science Meeting, San Antonio, TX, June 2002.

[13] S. S. Stuchly, C. L. Sibbald, and J. M. Anderson, "A new aperture admittance model for open-ended waveguides," IEEE Trans. Microwave Theory Tech., vol. 42, no. 2, pp. 192-198, Feb. 1994.

[14] E. T. Rosenbury, G. K. Burke, S. D. Nelson, R. D. Stever, G. K. Gorverno, and D. J. Mullenhoff, "Low cost impulse compatible wideband antenna," U. S. Patent 6348898.

[15] S. C. Davis, E. J. Bond, Xu Li, S. C. Hagness, and B. D. Van Veen, "Microwave imaging via space-time beamforming for early-stage breast cancer detection: Beamformer design in the frequency domain," $J$. Electromagnetic Waves and Applications, in press. 